\title{
On Power Measurements of Single-Electrode Low-Power Ar Plasma Jets
}

\author{
Vadym Prysiazhnyi ${ }^{1}$ (D) - Alonso H. C. Ricci ${ }^{1}$ - Konstantin G. Kostov ${ }^{1}$
}

Received: 17 April 2016 / Published online: 30 June 2016

(C) Sociedade Brasileira de Física 2016

\begin{abstract}
A study of electrical properties, methodology, and precision of power measurement was made on two types of $\mathrm{Ar}$ plasma jets, a single-strip-electrode plasma jet and a singlerod-electrode plasma jet. The dynamics of current peaks, methods for determining discharge power, and power measurement precision (especially important for applications in plasma medicine) are discussed for each type of plasma jet. Lower error in power calculation was obtained when the plasma jet did not touch the substrate, as well as more repetitive dynamics of the current peaks. Averaging high number of periods (over 500) when calculating the power by the Lissajous figure technique led to decrease of the experimental error.
\end{abstract}

Keywords Plasma jet - Electric characteristics $\cdot$ Power measurement

\section{Introduction}

Nowadays, small plasma jets exhibit a wide variety of applications such as polymer surface modifications [1], sterilization of bacteria [2], or dentistry applications [3]. Many research groups published reports about characterization of various homemade plasma jets. There are differences in the material of dielectric tube, geometry of electrodes, and operation parameters (applied voltage signal shape, working gas, etc.).

Vadym Prysiazhnyi

vadym.p@protonmail.com

1 Engineering Faculty of Guaratinguetá - FEG, State University of São Paulo - UNESP, Av. Dr. Ariberto Pereira da Cunha, 333, Guaratinguetá 12516-410, SP, Brazil
However, it is a rather complicated issue when one tries to compare different plasma jet arrangements. The parameters that can be used for comparison are dissipated power, plasma density, or plasma temperatures. In this work, we would like to focus on the methods for calculation of dissipated power measurement and their precision.

There are several ways to evaluate the consumed power in plasma jets. One is a power determination using Q-V plot (or Lissajous figures), when a capacitor is connected in series with the plasma jet and its charge is measured simultaneously with the applied voltage used for plasma generation. Due to a phase shift between these time variables, a closed figure is formed when, on one axis, the voltage applied to the jet and, on the other, the charge on the external capacitor, are plotted. The area of the figure is proportional to the power dissipated in the plasma jet [4]. Other method often reported in literature is a direct integration of voltage and current waveforms over one (or more) periods [5]. Though this method is reliable for determining power of plasma reactors with high power, it has not been considered good for low-power plasmas. A interesting way to measure the dissipated power was used in a work of Bruggeman et al. [6], when the power is calculated by measuring the temperature difference on the resistor connected in series with the plasma reactor when Ar flow is on and off. Other potential method of calculating the power is to estimate the charge transferred by the plasma directly, as it was proposed by Yambe et al. [7], where the authors estimate the charge of plasma bullets in He jet. Though the two above mentioned approaches are interesting, they require more sophisticated approach and could not be utilized as a fast tool.

Electrical characterization of plasma jets has been a subject of many works. For instance, a double-electrode Ar plasma jet that operates at AC frequency was developed by Nikiforov et al. [8]. In that research, the authors studied the influence of gas mixtures on optical and electrical characteristics. They showed that current 
waveforms of Ar plasma jet typically exhibit several current peaks (in positive and negative voltage semi-cycles), and that the consumed power is higher in the positive half periods. A higher applied voltage results to larger power consumptions [9] as more plasma is generated. Hong et al. [10] and Hao et al. [11] showed the relation between plasma jet power and applied voltage; however, they did not comment on precision of their measurements as well as how the measurements were influenced by the experimental setup geometry. The dependence of dissipated power versus applied frequency was reported in the work of Jin et al. [12], but there were no comments about the precision of these measurements.

We believe that there is one typical issue with reported data in the literature. Many authors do not provide a discussion about the precision of their power measurements. This issue was investigated for dielectric barrier discharge (DBD) plasma sources, as dissipated power is an important technological parameter [13]. It has been shown that one should be careful about power estimation, especially in the case of low-power plasma jets for bio-applications that are at a high interest of research community. We are not aware of similar studies that were performed for the plasma jets.

The goal of this article is to present the electrical characteristics of different single-electrode plasma jets together with a statistical study of the power calculations using two most utilized experimental approaches. The current peak dynamics depend on the jet type, and the precision of power determination will be discussed. Finally, we will give an insight about the most efficient and precise way to determine power in lowpower single-electrode plasma jets.

\section{Experimental}

\subsection{Plasma Sources}

Usually, plasma jets are produced and blown out from dielectric tubes complemented with metal electrodes. In this work, two types of tubes (alumina ceramic and Pyrex glass) and two types of metal electrodes (steel metal rod and aluminum adhesive tape) were used. The study of two principal singleelectrode jet configurations is presented: a single-stripelectrode plasma jet and a single-rod-electrode plasma jet. To eliminate numerous effects of gas mixture, it was decided to work only with analytical Ar (99.999\%) as a carrier gas. The flow was controlled by a rotameter Dwyer and was changed from 0.2 to $2 \mathrm{~L} / \mathrm{min}$. Most experiments were carried at gas flow of $0.8 \mathrm{~L} / \mathrm{min}$, because at this value, we get the longest plume length for the single-strip-electrode plasma jet.

In the first type of plasma jet, the plasma was generated inside a thin alumina tube with an outer diameter of $2.1 \mathrm{~mm}$ and an inner diameter of $1 \mathrm{~mm}$. High voltage was applied to an aluminum strip of 8-mm width wrapped around the tube at a distance of $10 \mathrm{~mm}$ from the nozzle. Second plasma jet was produced by inserting a sharp-edged 1.1-mm-thick metal rod into a Pyrex tube with an outer diameter $7.5 \mathrm{~mm}$ and an inner diameter of $3.3 \mathrm{~mm}$. The distance between the metal rod end and the jet nozzle is fixed to $40 \mathrm{~mm}$. High-voltage cable was connected to the metal rod. Figure 1 shows a typical view of plasma jet at operation.

\subsection{Power Supply}

The high voltage was generated by a commercial power supply Minipuls 4 (GBS Elektronik, Germany). It was developed to generate high $\mathrm{AC}$ voltages up to $40 \mathrm{kV}$ p-p with operation frequency from 5 to $40 \mathrm{kHz}$. The device consists of a full-bridge converter and a transformer cascade. Low voltages to the converter are supplied using the Voltcraft PS3620 laboratory power supply. The converter delivers a powerful low-voltage square wave, and the cascade transforms this up and filters out a sinelike waveform. The frequency of the signal was controlled by a signal generator Rigol DG1012. Due to a specific coupling between the transformer cascade and the plasma jet at frequency of $19 \mathrm{kHz}$, the output signal is a combination of two sine-like waves (one with higher amplitude and one with lower). Such mode is beneficial for our purposes, as it allows operating the plasma jet in semi-pulsed regime (when most of the plasma is generated in the higher-amplitude cycle). This effect contributes for the generation of cold plasma with less tube heating. Further in the text, these two sine-like signals will be referred as cycles. It was decided to use this mode of operation, as we had issues with heating and cracking of dielectric tubes when operating at higher frequencies with continuous wave signal.

\subsection{Electric Characterization}

Electric characterization of the plasma jets was performed by measuring the current and voltage waveforms, and power was evaluated by direct integration of voltage and current signals or Q-V plot/Lissajous figures [14]. The waveforms of current and

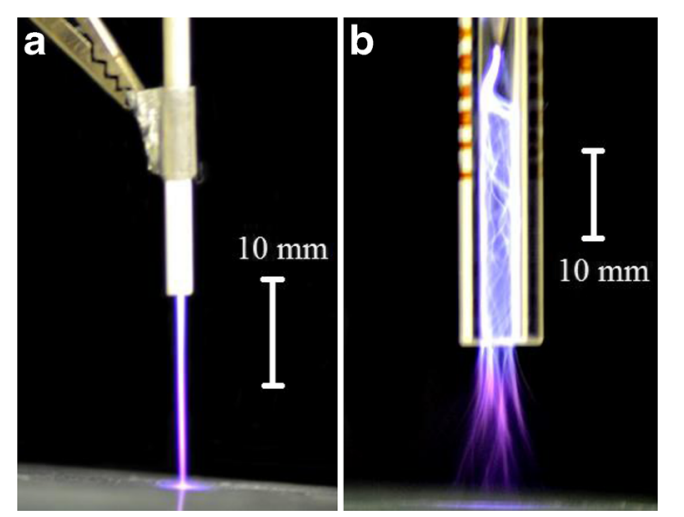

Fig. 1 Typical view of generated plasma: a single-strip-electrode plasma jet and $\mathbf{b}$ single-rod-electrode plasma jet for Ar gas flow of $0.8 \mathrm{~L} / \mathrm{min}$ and applied voltage of $15 \mathrm{kV}$ - $-\mathrm{p}$ 
voltage were recorded on Tektronix TDS 3032 oscilloscope (300 MHz, $2.5 \mathrm{GS} / \mathrm{s}$ ). High voltage was obtained from a voltage divider on the generator $(1: 2000)$. Current on a serial resistor (for direct integration) or voltage drop across an external capacitor (for Lissajous figures) was measured using a Tektronix P2220 probe. Depending on the measurement type, an inductance-free resistor of $104.7 \Omega$ or capacitor of $10 \mathrm{nF}$ was connected in series to a grounded stage. The stage consists of a 2.3-mm-thick glass of 150 -mm diameter with a 5-mm-thick metal plate with a diameter of $115 \mathrm{~mm}$ placed underneath. The glass is placed to prevent arc transition, while large metal size allows neglecting the edge effects while measuring the electric parameters. The schematic of experimental setup is shown in Fig. 2.

\subsection{Power Determination Techniques}

Two main experimental techniques are typically reported for calculation of dissipated power in electrical discharges. They are the direct integration technique and the method based on the Lissajous figure. Direct integration technique requires measurement of current and voltage waveforms from one or more periods. The dissipated power in the electric discharge can be evaluated by the following formula:

$P=f \cdot \int_{0}^{T} U(t) \cdot I(t) \cdot d t=f \cdot \sum_{i=1}^{N} U_{i} \cdot I_{i} \cdot \Delta t$,

where $f$ is the signal frequency, $T$ is its period, $N$ is the number of points in one period (for a digital oscilloscope), and $\Delta t$ is the sampling interval.

The power of electric discharge from Q-V plot is obtained using the phase shift between voltage applied to the plasma device $(V)$ and the transferred charge measured on a capacitor $(q)$ connected in series. If one plots $V(q)$ for one voltage cycle, the power can be calculated from the area of Lissajous figure by the following equation:

$P=f \cdot \oint_{T} V(q) \cdot d q=f \cdot[$ Area of the Lissajous figure $]$.

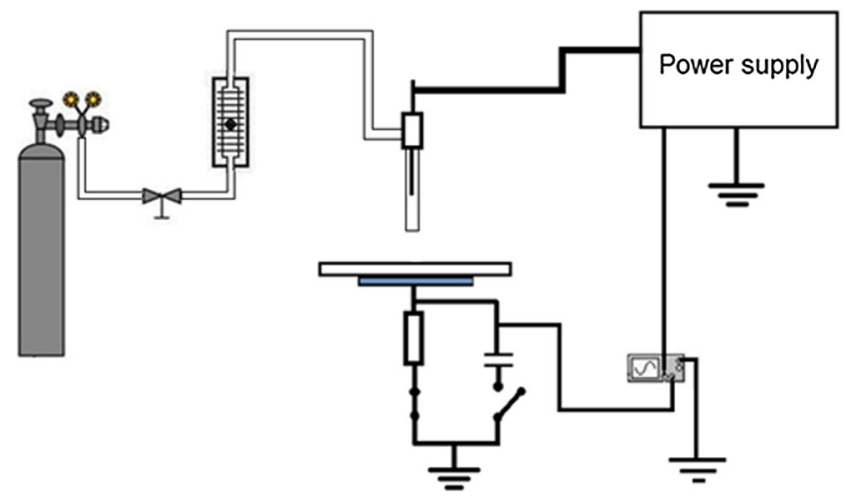

Fig. 2 Schema of experimental setup
Note that both methods give an overestimation of the dissipated power, since the voltage is measured at the voltage generator as in many other works [15]. An equivalent circuit of plasma jet is rather complicated [16] and depends on the electrode geometry (nowadays many different jet geometries exist [17]). Nevertheless, the main advantage of these methods is performing fast estimation of the input power.

\section{Results and Discussion}

\subsection{Current Waveforms}

Since the ceramic tube has a small inner diameter, the singlestrip-electrode plasma jet looks spatially uniform, forming a straight plasma column. However, after analyzing the photos taken with a conventional photocamera with short exposition time, we observed that the plasma plume consisted of several filaments that were located close to each other. The Reynolds number for Ar at a given geometry and gas flow $0.8 \mathrm{~L} / \mathrm{min}$ is about 1320. Until 2100, the flow can be considered laminar, which was observed with turned on plasma. The current waveforms exhibit three positive current peaks and one negative current peak in each high-amplitude cycle and one (sometimes two) positive current peak and one negative peak in the low-amplitude cycle, as shown in Fig. 3. If the plasma is touching the substrate (distance between the jet nozzle and the substrate less than $20 \mathrm{~mm}$ for gas flow of $0.8 \mathrm{~L} / \mathrm{min}$ ), the above-mentioned current peaks occur in each period. When the plasma does not touch the substrate (the distance is more than $25 \mathrm{~mm}$ for gas flow $0.8 \mathrm{~L} / \mathrm{min}$ ), the number of positive current peaks could be less or they do not appear in some periods. For large nozzle-substrate distances (more than $40 \mathrm{~mm}$ ) in the high-amplitude cycle, there was only one positive current peak, while in the low-amplitude cycle, the peaks were not present. It should be noted that here and below, the reference "touch the substrate" means that with lights off, it is possible to see that the plasma is in direct contact with the

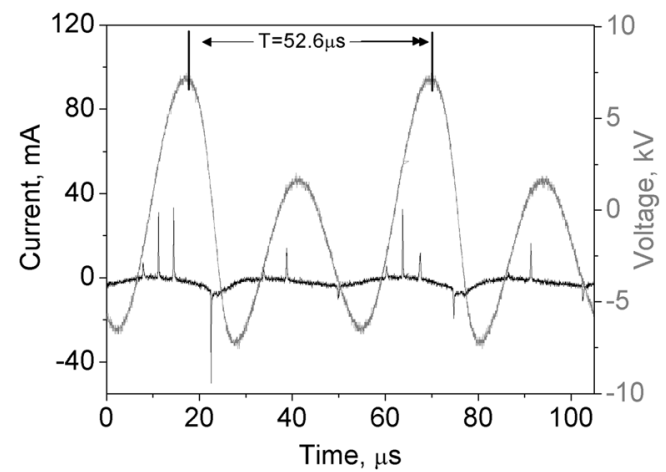

Fig. 3 A typical current waveform for the single-strip-electrode plasma jet: gas flow $0.8 \mathrm{~L} / \mathrm{min}$, voltage $15 \mathrm{kV} \mathrm{p}$-p, and nozzle-substrate distance $15 \mathrm{~mm}$ 
substrate by naked eye examination. When it is referred in the text "do not touch the substrate," it means that at the same light conditions, the gap between the tip of the plasma plume and the substrate can be seen by a naked eye. For a certain range of distances, there is a transition region, when, visually, not all but only some filaments reach the substrate. This case will be referred in the text as a "transition region" or "plasma slightly touching the substrate."

When jet is operated at low gas flows from 0.6 to $1.3 \mathrm{~L} /$ $\mathrm{min}$, the visual appearance of the single-rod-electrode plasma jet is of randomly distributed and spatially unstable showerlike filaments. Though the Reynolds number for gas flow of given jet geometry is 400 (flow should be laminar), Foletto et al. [18] confirmed that turning the plasma on can induce gas turbulence. Unlike with the first jet, larger inner diameter of the dielectric tube can be a reason to be perturbing low gas flow [19]. When the jet is operated at gas flows over $1.5 \mathrm{~L} /$ min, the discharge transforms into a bright single filament located in the tube center. Though, using a camera with short shutter times shows that those filaments are still present but pushed together by a gas flow dynamics. Note that the visual appearance of the single-rod-electrode plasma jet had more chaotic behavior in comparison with the single-stripelectrode plasma jet mentioned above; there were only two positive current peaks and one negative current peak in the high-amplitude cycle and one (or none in some periods) positive peak in the low-amplitude cycle, like it is shown in Fig. 4. Also, the current amplitude is lower and appearance of the current peaks is less often from one period to other. An increase of the substrate-to-nozzle distance (when the plasma does not touch the substrate) led to a decrease of the peak amplitude and their less frequent occurrence in each consequent period. The major difference between both jets is the amplitude of negative current peaks. For the single-stripelectrode plasma jet, the amplitude could be as high as for the positive peaks, while for the single-rod-electrode plasma jet, the negative peak amplitude was always lower than the amplitude of positive current peaks.

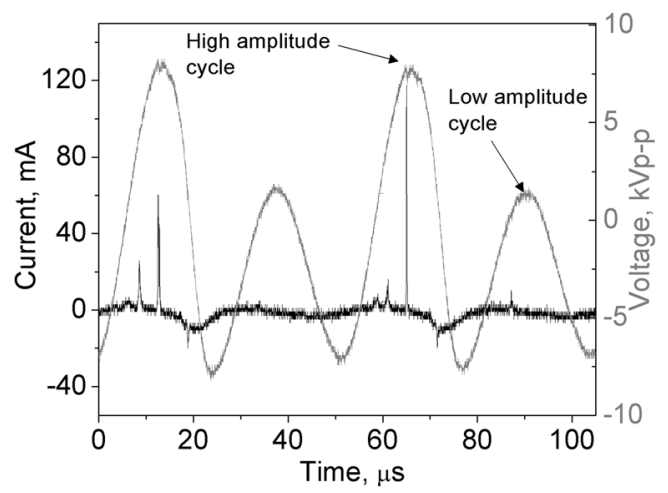

Fig. 4 A typical current waveform for the single-rod-electrode plasma jet: gas flow $0.8 \mathrm{~L} / \mathrm{min}$, voltage $15.5 \mathrm{kV} \mathrm{p}$-p, and nozzle-substrate distance $10 \mathrm{~mm}$

\subsection{Lissajous Figures}

It was confirmed by many measurements that the shape of the Lissajous figures for both plasma jets was quite similar. Unlike the regular parallelepiped-like shape of Q-V plots for DBD plasma, the figures are smoother and exhibit two closed shapes for each voltage cycle (larger loop corresponds to the higher-amplitude cycle and the smaller loop corresponds to the lower-amplitude cycle). Depending on the current peak number, the Q-V plot exhibits the same number of slope changes [20]. Figures 5 and 6 show the Q-V plots for the single-strip and single-rod plasma jets when the plasma is touching the substrate, respectively. These figures were obtained using averaging over more than 500 consequent periods. It can be done either manually or using the oscilloscope built-in function of averaging. It should be noticed that though visually, plasma of the single-strip plasma jet looks more stable than the plasma generated in the single-rod jet, the current peak positions and their presence during the discharge operation were more stable and repeatable for a single-rod plasma jet.

\subsection{Change of Power Depending on the Nozzle-Substrate Distance}

The studied plasma jets represent one-electrode system, which means that the presence of a substrate and its dielectric properties will strongly influence the way that the discharge operates. The plasma can be ignited when the jet is operated in a single-electrode mode (without a substrate), with a dielectric, with a dielectric with a floating or grounded metal located beneath, or with a conductive substrate. Each of these conditions influences the discharge operation as the configuration of the electric field will be different in each mentioned case. To simplify the system, a 2.3-mm-thick glass disc with dimensions $20 \mathrm{~cm} \times 20 \mathrm{~cm}$ was used as a substrate. Since a conductive electrode is necessary for power calculation using both

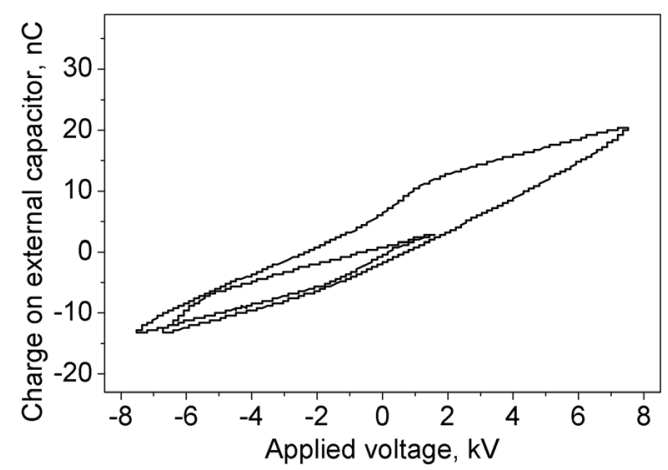

Fig. 5 Q-V plot for the single-strip-electrode plasma jet: gas flow $0.8 \mathrm{~L} /$ $\mathrm{min}$, nozzle-substrate distance $10 \mathrm{~mm}$, and applied voltage $15 \mathrm{kV}$ p-p 


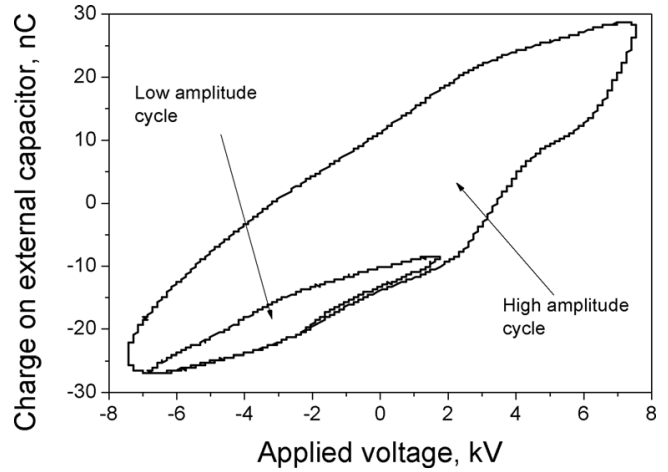

Fig. 6 Q-V plot for the single-rod-electrode plasma jet: gas flow $0.8 \mathrm{~L} /$ min, nozzle-substrate distance $10 \mathrm{~mm}$, and applied voltage $15 \mathrm{kV} \mathrm{p}$-p

methods, an aluminum disc with diameter of $115 \mathrm{~mm}$ was placed under the glass substrate. Due to the large size of the metal disc compared with the plasma jet, the edge effects that may influence the measurements can be neglected.

To calculate the input power using the Q-V plot, first, it is necessary to find out the area of the high- and low-amplitude cycles separately (see Fig. 6) and then add them. This will give the total area of the Lissajous figure for one period of voltage signal. Then, the power can be found by multiplying this area by the signal frequency, using Eq. (2).

Figures 7 and 8 represent the dependence of power on the distance between the jet nozzle and the glass substrate for the single-strip and the single-rod-electrode plasma jets. The measurements were performed under a high value of consequent period averaging 512 times and averaging over several trials for each experimental condition.

The difference in absolute values of power (maximum power did not exceed $2 \mathrm{~W}$ for the single strip and was up to $11 \mathrm{~W}$ for the single-rod plasma jets) is due to the different sizes of the plasma jets, i.e., different volumes of the generated plasma. Three regions can be distinguished for both jet types: the plasma jet does not touch the glass substrate (region C); the plasma jet is slightly touching the surface (region B), and

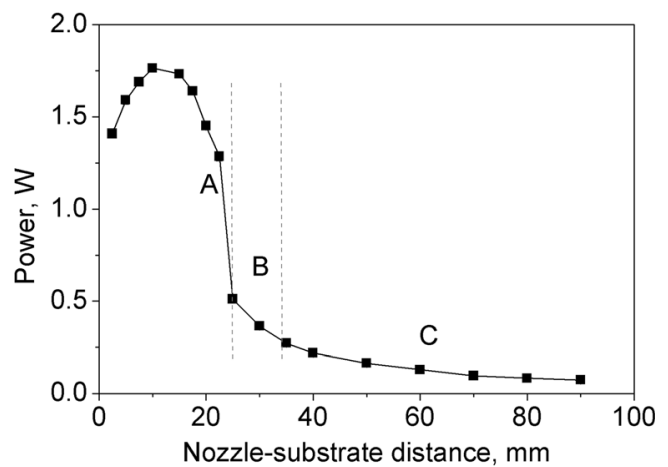

Fig. 7 Input power in a single-strip-electrode plasma jet as a function of the nozzle-substrate distance: gas flow $0.8 \mathrm{~L} / \mathrm{min}$, and voltage $15 \mathrm{kV}$ p-p. Error of the measurements is within $3 \%$ range

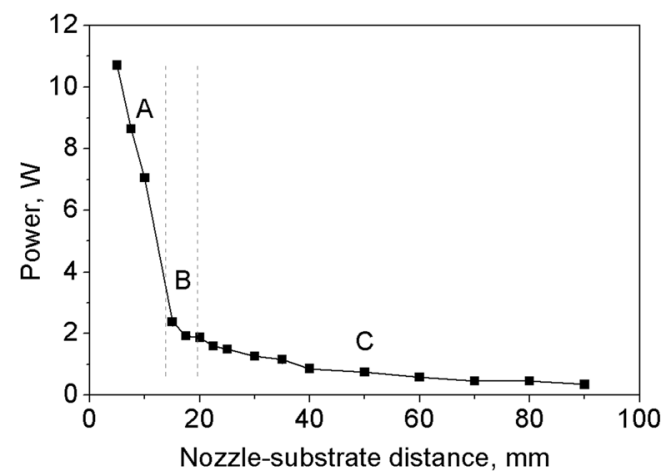

Fig. 8 Input power in a single-rod-electrode plasma jet as a function of the nozzle-substrate distance: gas flow $0.8 \mathrm{~L} / \mathrm{min}$ and voltage $15 \mathrm{kV}$ p-p. Error of the measurements is within $3 \%$ range

plasma jet touches the substrate (region A). The "touching" was confirmed by a naked eye when the plasma was ignited under the same external conditions (dark room). Due to different jet geometries, the absolute values of nozzle-substrate distances that define those regions are also different. For instance, a single-strip-electrode plasma jet has a length of about $25 \mathrm{~mm}$ for gas flow of $0.8 \mathrm{~L} / \mathrm{min}$, while a single-rod-electrode plasma jet length is about $16 \mathrm{~mm}$ for the same gas flow. It is obvious that the absolute values of these regions depend on a flow rate as the plasma plume length is flow dependent. For example, operating both plasma jets with lower flows led to shifting of the region borders to smaller nozzle/substrate distances. The borders of the regions are marked on Figs. 7 and 8 . The plasma touches the substrate for distance less than $25 \mathrm{~mm}$ and does not touch the substrate for distances more than $30 \mathrm{~mm}$ for the single-strip-electrode plasma jet. When the distance is between 25 and $30 \mathrm{~mm}$, some filaments are reaching the substrate, but not all of them. For the singlerod-electrode plasma jet, those distances are 16 and $22 \mathrm{~mm}$, respectively.

The power dissipated in the plasma jet for region $\mathrm{C}$ (when the plasma jet is far from the glass substrate) tends to decrease slowly until saturation due to the decreasing influence of the substrate.

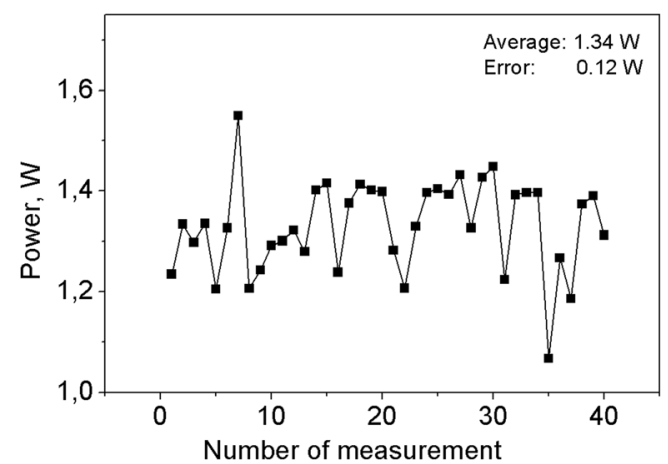

Fig. 9 Power obtained from 40 independent measurements using the direct integration technique for the single-rod-electrode plasma jet: nozzle-substrate distance $35 \mathrm{~mm}$, gas flow $0.8 \mathrm{~L} / \mathrm{min}$, and applied voltage $15 \mathrm{kV}$ p-p 
Table 1 Results of statistical study of the single-strip-electrode plasma jet

\begin{tabular}{lllll}
\hline & Region & Condition of measurement & Average power (W) & Experimental error (\%) \\
\hline \multirow{2}{*}{ Integration } & Region A & SW & 2.4 & 10 \\
& Region B & SW & 0.6 & 14 \\
& Region C & SW & 0.4 & 8 \\
Q-V plot & Region A & SW/128A/512A & $1.9 / 2.0 / 2.2$ & $14 / 1 /<1$ \\
& Region B & SW/128A/512A & $0.5 / 0.5 / 0.5$ & $20 /<1 /<1$ \\
& Region C & SW/128A/512A & $0.4 / 0.4 / 0.4$ & $13 / 1 /<1$ \\
\hline
\end{tabular}

SW single waveform, $128 \mathrm{~A}$ built-in oscilloscope averaging function over 128 periods, $512 \mathrm{~A}$ built-in averaging function over 512 periods, region $A$ nozzle-substrate distance $15 \mathrm{~mm}$, region $B$ nozzle-substrate distance $25 \mathrm{~mm}$, region $C$ nozzle-substrate distance $40 \mathrm{~mm}$
The power starts increasing more rapidly, when the plasma column is in contact with the substrate due to the effect of capacitive coupling. This corresponds to regions B and A. It can be seen by the slope change in the power versus distance dependence. For distances less than $10 \mathrm{~mm}$ in the case of a single-strip-electrode plasma jet, it was observed that the consumed power decreased. This effect can be attributed to the interference of the gas flow dynamics on the jet operation. Using the Schlieren imaging, Robert et al. [21] and Boselli et al. [22] showed that the plasma is affected by the gas flow. For very small nozzle-to-substrate distances, the flow will transform to strongly turbulent, thus preventing the formation of larger volume of plasma.

\subsection{Statistics of the Measurements}

In the next set of experiments, we examine the scattering of the consumed power obtained from $\mathrm{Q}-\mathrm{V}$ plot and direct integration techniques for the previously defined regions $\mathrm{A}, \mathrm{B}$, and $\mathrm{C}$. To study the stability of power calculations, it was decided to record 40 waveforms of applied voltage/charge on capacitor or applied voltage/current through the external resistance for a single set of operation parameters. These 40 waveforms were used to evaluate the dissipated power and the error of the power estimation. The oscilloscope built-in function of data averaging (simple geometrical averaging of operator-defined consequent periods) was used for the Lissajous figure method to check the stability of this technique. An example of one set using the direct integration is presented on Fig. 9 for the single-rod-electrode plasma jet operated in the region $\mathrm{C}$ when the nozzle-to-substrate distance was $35 \mathrm{~mm}$. The results for both jets (the single-strip and the single-rod plasma jets) are summarized in Tables 1 and 2 .

The statistical analysis of the power measurements showed several features. Both experimental techniques can be used to calculate the input power in single-electrode plasma jets. The best agreement between both techniques is obtained in region $\mathrm{C}$ when the plasma plume does not touch the substrate as the jet is the most stable (by visual observation also). Lowest error in power evaluation was obtained using direct integration technique for the high nozzle-to-substrate distances (region C). In this case, the integration technique can be also used, especially when direct contact between the plasma and the sample is not necessary (or harmful in case of high thermal sensitivity of surface) and plasma is used only to create radicals that will reach the substrate carried by the gas flow.

When the Q-V plot method is used, it is necessary to use averaging over a large number of periods to get more precise result. It is possible either to do it manually or to use the average function of the oscilloscope, which makes the measurements fast. For both jets, the power calculation using the Lissajous figure technique is giving reasonably low data scattering (less than $4 \%$ ) when the averaging of more than 100 periods is used.

In the transition region (region B), when filaments start touching the substrate, one should be careful as we found that for both types of jets, there is a higher data scattering in the power obtained from the direct integration technique (14 and
Table 2 Results of statistical study of the single-rod-electrode plasma jet

\begin{tabular}{lllll}
\hline \multirow{4}{*}{ Integration } & Region & Condition of measurements & Average power (W) & Experimental error (\%) \\
& Region A & SW & 5.9 & 15 \\
& Region B & SW & 1.8 & 16 \\
& Region C & SW & 1.3 & 9 \\
& Region A & SW/128A/512A & $5 / 6.6 / 6.7$ & $23 / 1 / 1$ \\
& Region B & SW/128A/512A & $1.7 / 1.8 / 1.8$ & $16 / 2 / 2$ \\
& Region C & SW/128A/512A & $1.35 / 1.3 / 1.3$ & $9 / 3 / 2$ \\
\hline
\end{tabular}

region $A$ nozzle-substrate distance $10 \mathrm{~mm}$, region $B$ nozzle-substrate distance $19 \mathrm{~mm}$, region $C$ nozzle-substrate distance $30 \mathrm{~mm}$ 
$16 \%$ for the single-strip and single-rod plasma jets). The increased instability of the transition region is probably related to the fact that not all filaments are reaching the dielectric substrate. This leads to reduced charge on the substrate, making the filament formation in the next period less probable, similar to the case of microdischarge dynamics in DBDs [23, 24]. In this case, the use of direct integration should be done with a special care and the preferred technique should be power calculation using averaged Q-V plot. However, the most common case, i.e., when plasma is used for surface treatments, the input power should be calculated from the Q-V plot averaged over many periods. This is especially important for the case of low-power plasma sources, when spatially/temporarily unstable plasma can lead to an underestimation or overestimation of the total power.

\section{Conclusions}

The electrical properties of two single-electrode plasma jets operated in pure Ar were investigated. It has been shown that even though visually more stable, the plasma generated in a tube with a smaller diameter does not have well-defined and reproducible current peaks. The visual appearance of plasma strongly depends on the gas flow, which is especially important when surface treatments are considered.

Two methods of power calculation for single-electrode plasma jets were compared. It was shown that the power evaluation is greatly influenced by the experimental configuration, in this case, by the distance between the jet nozzle and the glass substrate. This distance must be taken with great caution in the case when the plasma is touching the substrate, because the power values can significantly increase when the distance is changed only slightly. For example, the change from 10 to $7.5 \mathrm{~mm}$ leads to change of input power from 6.7 to $8.3 \mathrm{~W}$ for the single-rod-electrode plasma jet. The precision of the power calculation was investigated for three principal conditions (the separation between them is the plasma visual appearance): when plasma does not touch the substrate, slightly touching it, and in a contact with the substrate.

The direct integration technique should be used carefully, as high data averaging is necessary (hundreds of periods). Also, even visually, stable small plasmas can have high uncertainty in power determination because of their filamentary nature, which should be always taken into a consideration. Through the analysis of the position, amplitude, and appearance of the current peaks, additional information about the discharge dynamics of the current filaments can be gained from the direct integration technique.

The lowest experimental error in calculation of input power for both jet types is obtained by $\mathrm{Q}-\mathrm{V}$ plot with high number of periods for averaging. Using this technique, one can get an experimental error in the range of $3 \%$ or less. To obtain measurements in a short time, it is possible to use the oscilloscope built-in average function (simple average of $\mathrm{X}$ consequent waveforms).

Acknowledgments This research was supported by Fundação de Amparo à Pesquisa do Estado de São Paulo (FAPESP; grant No. 2013/ 06732-3) and Conselho Nacional de Desenvolvimento Científico e Tecnológico (CNPq; project No. 470995/2013-0).

\section{References}

1. M. Noeske, J. Degenhardt, S. Strudthoff, U. Lommatzsch, Int. J. Adhes. Adhes. 24, 171 (2004)

2. S. Ikawa, K. Kitano, S. Hamaguchi, Plasma Process. Polym. 7, 33 (2009)

3. H.W. Lee, G.J. Kim, J.M. Kim, J.K. Park, J.K. Lee, G.C. Kim, J. Endodont. 35, 587 (2009)

4. H. Jiang, T. Shao, C. Zhang, W. Li, P. Yan, X. Che, E. Schamiloglu, IEEE T. Dielect. El. In. 20, 1101 (2013)

5. Y. Jin, C.-S. Ren, Q.-Q. Fan, H. Yan, Z. Li, J. Zhang, D. Wang, IEEE T. Plasma Sci. 40, 2706 (2012)

6. S. Hoffmann, A.F.H. van Gessel, T. Verreycken, P. Bruggeman, Plasma Sources Sci. T. 20, 065010 (2011)

7. K. Yambe, H. Saito, K. Ogura, IEEJ Trans. Elec. Electron. Eng. 10, $614(2015)$

8. A.Y. Nikiforov, A. Sarani, C. Leys, Plasma Sources Sci. T. 20, 015014 (2011)

9. G.-D. Wei, C.-S. Ren, M.-Y. Qian, Q.-Y. Nie, IEEE T. Plasma Sci. 39, $1842(2011)$

10. Y. Hong, N. Lu, J. Pan, J. Li, Y. Wu, K.F. Shang, J. Electrostat. 71, $93(2013)$

11. Z. Hao, S. Ji, H. Liu, Y. Song, IEEE T. Plasma Sci. 42, 824 (2014)

12. Y. Jin, C. Ren, L. Yang, J. Zhang, D. Wang, Plasma Sci. Technol. 15, 1203 (2013)

13. J. Kriegseis, B. Moller, S. Grundmann, C. Tropea, J. Electrostat. 69, $302(2011)$

14. M. Hołub, Int. J. Appl. Electrom. 39, 81 (2012)

15. Z. Fang, J. Yang, Y. Liu, T. Shao, C. Zhang, IEEE T. Plasma Sci. 41, 1627 (2013)

16. H. Akamatsu, K. Ichikawa, Surf. Coat. Technol. 206, 920 (2011)

17. X. Lu, M. Laroussi, V. Puech, Plasma Sources Sci. Technol. 21, 034005 (2012)

18. M. Foletto, C. Douat, J. Fontane, L. Joly, L. Pitchford, V. Puech, Influence of a plasma jet on the hydrodynamics of a helium jet, in 31th International Conference on Phenomena in Ionized Gases ICPIG, 14-19 Jul 2013, Granada, Spain, 2013

19. Z. Xiong, E. Robert, V. Sarron, J.-M. Pouvesle, M.J. Kushner, J. Phys. D. Appl. Phys. 46, 155203 (2013)

20. F. Liu, W. Wang, X. Chang, Z. Wu, L. He, Z. Li, Z. He, R. Liang, EPL (Europhysics Letters) 97, 65001 (2012)

21. E. Robert, V. Sarron, T. Darny, D. Ries, S. Dozias, J. Fontane, L. Joly, J.-M. Pouvesle, Plasma Sources Sci. T. 23, 012003 (2014)

22. M. Boselli, V. Colombo, E. Ghedini, M. Gherardi, R. Laurita, A. Liguori, P. Sanibondi, A. Stancampiano, Plasma Chem. Plasma Process. 34, 853-869 (2014)

23. S. Celestin, G. Canes-Boussard, O. Guaitella, A. Bourdon, A. Rousseau, J. Phys. D. Appl. Phys. 41, 205214 (2008)

24. M. Bogaczyk, R. Wild, L. Stollenwerk, H.-E. Wagner, J. Phys. D. Appl. Phys. 45, 465202 (2012) 